\title{
A decalogue for end-of-life care in Internal Medicine
}

\author{
Luigi Lusiani, ${ }^{1}$ Giorgio Ballardini, ${ }^{2}$ Roberto Nardi, ${ }^{3}$ Luigi Magnani, ${ }^{4}$ Claudio Santini,,${ }^{5}$ Giovanna Pentella, ${ }^{6}$ \\ Andrea Fontanella, ${ }^{7}$ on behalf of the FADOI-ANIMO Committee for the End-of-Life Care in Internal Medicine* \\ ${ }^{1}$ Department of Internal Medicine, S. Giacomo Hospital, Castelfranco Veneto (TV); ${ }^{2}$ Department of Internal Medicine, Infermi \\ Hospital, Rimini; ${ }^{3}$ Internal Medicine, Bologna; ${ }^{4}$ Department of Internal Medicine, Hospital of Voghera, ASST Pavia; \\ ${ }^{5}$ Department of Internal Medicine, Madre Giuseppina Vannini Hospital, Figlie di S. Camillo, Roma; ${ }^{6}$ Dirigenza Infermieristica, \\ Fatebenefratelli Hospital, Napoli; ${ }^{7}$ Internal Medicine Unit, Fatebenefratelli Hospital, Napoli, Italy
}

\begin{abstract}
Since a large number of patients with chronical medical diseases die in hospital, often in an internal medicine ward, internists are urged to improve their expertise in end-of-life (EOL) care, which is a neglected part of their academic education. Recently, FADOI (the Italian Federation of the Associations Hospital Doctors on Internal Medicine) has addressed EOL-medicine in many ways, promoting many scientific meetings on this and allied topics, providing educational material made available in its website on a free basis and establishing an ad hoc Committee charged with the task of organizing dedicated events annually. The Committee has also elaborated a series of recommendations on EOL-care in internal medicine (a decalogue), reflecting largely shared visions. It has been endorsed also by ANIMO (the Association of the Italian Nurses working in an Internal Medicine Department). The decalogue for EOL care in internal medicine is issued here, and calls for its diffusion and implementation. The driving concept is that doctors and nurses must feel responsible for disregarding appropriate EOL-care for the dying patients, because delaying it means to add suffering and discomfort to them in the final phase of their existence.
\end{abstract}

\section{End-of-life medicine}

Patients with chronic medical disease in Italy express their preference to conclude their life at home, hopefully surrounded by a familiar and comfortable environment. ${ }^{1,2}$ And yet, as a matter of fact, a large

Correspondence: Luigi Lusiani, Department of Internal Medicine, S. Giacomo Hospital, via dei Carpani, 16/Z, 31033 Castelfranco Veneto (TV), Italy.

E-mail: g.lusiani52@gmail.com

Key words: End-of-life care.

Conflict of interest: the authors declare no potential conflict of interest.

*The FADOI-ANIMO Committee for the End-of-Life Care in Internal Medicine: Mauro Carbone, Giuseppe Chiesi, Giuseppe Civardi, Luigino Di Napoli, Mario Felici, Roberto Frediani, Fabio Giglioli, Valentino Moretti, Domenico Panuccio, Giuliano Pinna, Roberto Risicato (FADOI) and Lorella Rossetti (ANIMO).

Received for publication: 19 June 2018.

Revision received: 20 June 2018

Accepted for publication: 22 June 2018.

This work is licensed under a Creative Commons Attribution NonCommercial 4.0 License (CC BY-NC 4.0).

CC Copyright L. Lusiani et al., 2018

Licensee PAGEPress, Italy

Italian Journal of Medicine 2018; 12:230-234

doi:10.4081/itjm.2018.1060 number of them die in hospital, often in an internal medicine ward, ${ }^{3,4}$ affected by single or multiple endstage organ failures, at the end of trajectories characterized by repeated hospital admissions, due to acute worsening or intervening complications or, more simply, to further deterioration of their condition that makes home care inconvenient. ${ }^{5,6}$ In this kind of patients (elderly, with multiple diseases and irreversible organ failures), the death is easily anticipated, even though a substantial uncertainty about its timing always exists, due to the lack of accurate prognostic indexes. ${ }^{7,8}$

For example, out of the total number of 198,156 deaths registered in hospitalized patients during 2014 in Italy, 102,498 (or 51.7\%) occurred in Internal Medicine or Geriatric Departments, and those occurred specifically in Internal Medicine outnumbered all the others occurred in non-surgical departments, by a factor of 2.5 compared with Intensive Care Units, 5.6 with Geriatric, 7.9 with Cardiology, 2.5 with all the other medical specialties taken together (unpublished data, made available by Giovanni Mathieu).

These figures are not expected to vary in absolute terms in the future, because the increasing number of the aged people will counterbalance the availability of alternative care settings preventing hospitalization (such as hospices and other forms of home care, which, however, tend to be restricted to a subset of cancer patients).

These data demonstrate the necessity for internists, unavoidably involved in taking care of dying people, 
to improve their expertise in end-of-life (EOL) medicine, which has already become a consistent portion of their job. Unfortunately, generally speaking, internists have not received any specific and formal training on this topic (palliative medicine, comfort medicine), due to the fact that the academic teaching lags behind. ${ }^{9}$ Admittedly, valuable EOL-care remains an unmet need in most internal medicine departments. Time has come for addressing these issues more energetically.

\section{The FADOI strategy}

In the last few years, FADOI (the Italian Federation of Associations of Hospital Doctors on Internal Medicine) has addressed EOL-medicine in several ways, promoting many scientific meetings on this and allied topics, providing teaching materials on pain treatment and communication skill, and editing dedicated issues (Quaderni - Italian Journal of Medicine 2017, volume 5, issues 4 and 5). The full list of such activities is visible on its website, online (http://www.fadoi.org). Many FADOI members and followers have been allowed to access this educational program, made largely available on a free basis.

More recently (December 2017) an ad hoc committee of fourteen senior members has been established by the national FADOI board, in order to promote the organization of a one-day scientific meeting on EOL-care in Internal Medicine, to be held with the same format, on the same date all around Italy, in every single region or aggregated regions. This multi-site, simultaneous event took place on April 7, 2018, when a total number of about 1200 participants (both doctors and nurses) convened in the different meeting sites (17 in total), focusing on EOLmedicine.

The ad hoc committee was also charged with the task to elaborate a series of recommendations to be addressed to the largest audience of the Italian internists and internal medicine nurses, regarding the principles that must support the EOL-care in an internal medicine department. This collection of recommendations took the form of a decalogue, which was proposed at each regional meeting on April 7, 2018, for revision and approval. The final version received the contribution and the endorsement of the ANIMO (the Association of the Italian Nurses working in an Internal Medicine Department), and now a FADOI-ANIMO Decalogue for EOL-care in Internal Medicine is available for all doctors and nurses working there (Table 1). It is supposed to inspire and sustain their approach to the dying patients, reflecting largely shared visions.

During the latest society national congress (XXXIII
Congresso Nazionale FADOI-XIII Congresso Nazionale ANIMO, Bologna, May 12-14, 2018), the Decalogue was submitted to all participants (1635 doctor, 279 nurses). They were asked to rank each item of the Decalogue from 1 to 10 , in terms of importance (answering the following question: How important is the recommendation in your opinion?) and feasibility (How does the recommendation correspond with your practice?). The response rate was $14 \%$. The average scores ranged from 8.02 to 9.55 as for the importance issue, and from 6.92 to 8.20 as for the feasibility issue (with a maximum delta for the item No. 8, regarding the start of the palliative care). Although this survey was launched for awareness purposes, it can be interpreted as showing that, while recommendations are considered as highly important and are agreed upon by doctors and nurses, their implementation is far from being fulfilled in the everyday practice.

\section{Implementation}

These recommendations call for their implementation, which might take place through sequential steps.

The first step should regard the education of doctors and nurses, with the aim of expanding, strengthening and then maintaining the basic skills requested for a proficient EOL-care, namely, communication competence, pain treatment, artificial nutrition and palliative sedation, according to the principles of the comfort medicine. ${ }^{10}$ Hopefully, all Internists and Nurses working in an Internal Medicine department should be reached and exposed to such educational program and trained accordingly in the next few years.

The second step should deal with the organization of appropriate care setting and team work, within each internal medicine department. EOL-care is not only a matter of personal attitude and individual skill, but also of re-arrangement of room spaces, of redistribution of human resources and of rendering available other facilities. It requires doctors and nurses to become capable to decide together and to interact harmoniously. While the doctor component is crucial as for the decision to start, which needs to be based on a judicious clinical assessment, the nurse component plays the main role as for the conduction of the EOLcomfort medicine, which asks for continuative and pro-active bedside surveillance. It should be underlined that, basically, such organization should not necessarily require adjunctive resources.

The third step should be concerned with the objective evaluation of the results, in terms of actual degree of pain control in the dying patients, relief of other relevant symptoms, reduction of the number of 
Table 1. FADOI-ANIMO decalogue for end-of-life care in Internal Medicine.

1. Be aware of the problems associated with the end of life

- The availability of diagnostic tools and even more advanced treatments means that today an increasing number of patients die in conditions of unwarranted medicalization, without any benefits or improvement in the quality of the final stages of their lives

- It is not easy to define life expectancy and the beginning of the terminal phase of a disease, especially in non-cancer patients with chronic progressive diseases. This is because for these patients there are no validated and easyto-use tools to correctly estimate the prognosis

- The majority of deaths, whether they have been predicted or not, take place in the hospital, more often in Internal Medicine wards

- In the final stages of life there is a real risk of carrying out diagnostic investigations and treatments which are useless, if not actually harmful (overdiagnosis, overtreatment), not only in patients with a very short life expectation (days or weeks)

- Many patients (and their relatives/caregivers) who are hospitalized in the terminal stage of illness in Internal Medicine wards (especially if suffering from chronic non-oncological diseases) have never been properly informed about the severity of the prognosis or the fact that life expectancy is limited - In end-of-life patients admitted to internal medicine wards, medical and nursing practice is still insufficiently oriented towards palliative care and the treatment of terminal disease; instead, it remains focused on the treatment or the illness itself rather than on the control of the symptoms which undermine the quality of the patient's remaining days

- The shortcomings in this area are also brought about by inadequate organizational arrangements and attitudes, which neglect to facilitate interaction between the various health professions

2. Always strive to abide by the principles of the Codes of Medical Ethics 2016 and Nursing Ethics 2009 and respect the law regarding informed consent and advanced treatment provisions 219/17
In particular, with reference to the following articles:

- (medical code, Article 14) doctors must refrain from continuing with treatments from which a benefit to the quality of life cannot be justifiably expected

- (medical code, Article 17) doctors, even if requested by the patient, must not carry out or assist with treatments designed to cause death

- (medical code, Article 23) doctors cannot abandon any patient considered incurable, but must continue care, even if it is only to alleviate physical and mental suffering

- (nursing code, Article 34) nurses must seek to prevent and combat pain and to alleviate suffering... using all the necessary treatments

- (nursing code, Article 35) nurses must provide assistance whatever the clinical condition and until the end of the patient's life, recognizing the importance of palliation and environmental, physical, psychological, relational and spiritual comfort

- (nursing code, Article 36) nurses must protect the will of the patient to limit interventions which are not suitable for his/her clinical condition or consistent with his/her conception of quality of life

- (nursing code, Article 38) nurses must not pursue or take part in interventions aimed at causing the patient's death

- (nursing code, Article 39) nurses must support the relatives and loved ones of the patient, in particular in the final stages of the disease, at the moment of the patient's passing and during the mourning period

- (law 219/17 Article 2) doctors must refrain from any unreasonable persistence in the administration of treatment and from the use of unnecessary or excessive treatments

- (law 219/17 Article 4) everyone... can, through the Advance Provisions of Treatment, express their will with regard to health care

- (law 219/17 Article 5) the patient and, with his/her consent, his/her family members... must be adequately informed... on the possible progress of the disease, on what can be realistically expected in terms of quality of life, on the clinical possibilities of intervention and on palliative care

Treatment should be suited to the realistic prospects of the time the patient has left, using clinical judgement (often difficult) and completely respecting the will of the patient, no matter what it is, whilst taking into account his/her expectations (see Law 219/17). The requests of family members/caregivers must also be considered when they sincerely (that is, lovingly) support the patient's point of view and the reasoning behind it. Doing everything possible in situations of terminal illness shall be considered wrongful practice and shall be forbidden. Any clinical care (diagnostic, therapeutic and assistance) must be undertaken only if it is beneficial to the patient

To be continued on next page 
Table 1. Continued from previous page.

4. Care for terminal patients right to the end

To treat means not only to heal, but also (and especially in the terminal stages of an illness) to relieve suffering and a feeling of abandonment. The statement there is nothing left to be done when referring to the end-of-life patients is wrongful and should be forbidden. Instead, all possible forms of palliative care are to be guaranteed to the patient

5. Fight physical pain using all available means

Pain always undermines and jeopardizes the meaning of life and should not be considered unavoidable at the end of life. Health-care providers must therefore do their utmost to treat pain as effectively as possible, in line with the provisions of Law 38/2010 and the WHO recommendations

6. Promote special skills in artificial nutrition and hydration

Skills in artificial nutrition and hydration in terminally ill patients (effectively forms of treatment, as stipulated in Article 1 of Law 219/17) concern the guidelines, administration techniques and the assessment of needs, keeping in mind that:

- malnutrition ceases to be a predominant preoccupation at the end of life

- nutritional support in terminal patients at risk of malnutrition does not increase survival

- artificial nutrition can pose a risk of complications which can actually hasten death

- not placing a nasogastric tube or PEG for enteral feeding in the terminal patient does not hasten death and is not considered euthanasia

- the real benefits obtained from artificial nutrition and hydration must be carefully and practically evaluated in each individual patient, regardless of the symbolic value attributed to these methods, which have been elevated to constitute an absolute and unquestionable right (no one should be left to die of hunger or thirst)

- the customization of choices is the cornerstone of decision making

7. Promote special skills on palliative sedation

Medical staff must be prepared to resort to palliative sedation (intentional reduction of alertness using pharmacological means) for the control of refractory symptoms, as a tool to improve the quality of remaining life (as stated in Article 2 of Law 219/17: In the presence of refractory suffering during medical treatments, the doctor can resort to continuous deep palliative sedation in association with pain therapy, with the consent of the patient). The conceptual confusion between palliative sedation and euthanasia should be avoided

8. Do not delay the start of palliative care

In each individual case it is necessary to decide (based on a clinical judgment formulated with the utmost care, considering all available information and in accordance with scientific knowledge and experience) when active treatments, including life support, powerful as they are, no longer benefit the patient. It is morally correct not to implement treatments or to suspend them if they do not meet the ethical and humanist criterion of proportionality; this does not mean causing death, but accepting that it cannot be prevented

9. Be people as well as medical staff

Doctors and nurses must be absolutely able to utilize not only their scientific skills and technical abilities, but also - and ultimately predominantly - the ability to be human before another human who is suffering thus directly face the patient's unceasing dignity

10. Constantly maintain intense communication

Communication with terminally ill patients and their families/caregivers must be appropriate to their sensitivity and awareness, respectful of their spiritual needs and delicate in timing and manner. It should be based on understanding, sharing information and scientific knowledge according to which choices must be evaluated, in order to guarantee an overall approach oriented to the dignity of the patient

examinations and futile treatments during the last days, along with the degree of satisfaction of the care-giver and the health care personnel. Such evaluation can compare EOL-care with treatment as usual.

The internists and their nurses should be highly motivated not to delegate EOL-care to external specialists or dedicated teams, which, numerically insufficient as they are, could hardly take charge of such a relevant burden, neither at the present time, nor in a future perspective. ${ }^{11}$

\section{Conclusions}

Delaying appropriate EOL-care for the dying patients means to add suffering and discomfort to 
them in the final phase of their existence. Usual care not only tends to charge the patients with procedures and treatments from which they can hardly benefit, but also deviates doctors and nurses from what really matters to a patient at the end of his/her life, that is the relief of the disturbing symptoms. ${ }^{12}$

In no way EOL-medicine means giving $u p$ or letting go ${ }^{13}$ rather, it means to choose what is the best for the dying patients and to accomplish it, in a holistic, comprehensive and deeply respectful manner.

\section{References}

1. Beccaro M, Costantini M, Giorgi Rossi P, et al. Actual and preferred place of death of cancer patients. Results from the Italian survey of the dying of cancer (ISDOC). J Epidemiol Community Health 2006; 60:412-6.

2. Bertolissi S, Miccinesi G, Giusti F. Come si muore in Italia. Storia e risultati dello studio Senti-MELC. Rivista SIMG 2012;2:17-34.

3. Van der Heide A, Desmedt D, et al. Palliative inpatients in general hospital: a one-day observation study in Belgium. BMJ Palliative Care 2011;10:2.

4. Teno JM, Gonzalo PL, Bynum JP, et al. Change in the end-of-life care for Medicare beneficiaries: site of death, place of cares and health care transition in 2000, 2005 and 2009. JAMA 2013;309470-7.

5. Lorenz K, Lynn J, Sydney M, et al. Evidence for improving palliative care at the end of life: a systematic review. Ann Intern Med 2008;147:148-159.

6. Gill TM, Gahbauer EA, Han L, Allore GH. Trajectories of disabilities in the last year of life. N Engl J Med 2010;362:1173-80.

7. Nardi R, Belmonte G, Gnerre P, et al. Metodi di riconoscimento e di valutazione del paziente in fase terminale $\mathrm{o}$ a rischio di elevata mortalità in ospedale: le malattie più frequentemente riscontrabili in Medicina Interna. Quaderni - Italian Journal of Medicine 2017;5: 104-148.

8. Yourman LC, Lee SJ, Schonberg MA, Widera EW, Smith AK. Prognostic indices for older adults. A systematic review. JAMA 2012; 307:182-92.

9. Borasio GD. Saper morire. Torino: Bollati Boringhieri Ed., 2015.

10. Blinderman $\mathrm{CD}$, Billings AB. Comfort care for patients dying in the hospital. N Engl J Med 2015;373:2449-61.

11. Lupu D. Estimate of current hospice and palliative medicine physician work force shortage. J Pain Symptom Manage 2010;40:899-911.

12. Kelly AS, Morrison RS. Palliative care for the seriously ill. N Engl J Med 2015; 373:747-55.

13. Gawande A. Quantity and quality of life: duties of care in life-limiting illness. JAMA 2016;315:267-9. 